\title{
ELECTROMYOGRAPHIC INVESTIGATION \\ OF ESOPHAGUS, IN ANIMALS
}

\author{
* Tetsuzo INOUE, M. D.
}

\section{TABLE OF CONTENTS}

I Introduction with review articles

II Apparatus, Equipment and Materials

III Results

V Discussion

VI Conclusion and Summary

VII Bibliography

\section{Introduction}

Many investigators have studied the anatomy and physiology of the esophagus.

However, very little has been done with regad to electrophysiology of the esophagus (21).

The esophagus is the organ for deglutition which cnosists of two muscular layers with muscularis mucosa and mucous membrane. Histologically, it consists of striated muscle in the upper part of the organ and smooth muscle located in the lower part of the organ. The mid-esophagus is a mixture of both types, and the proportion varies from one individual to the other. This was described dy Lerche ${ }^{(18)}$.

Branches of the vagus nerve and cervical sympathetic fibers innervate the cervical esophagus, and vagal and thoracic sympathetic fibers go to the thoracic esophagus(11,16).

The tetanic contraction of esophagus was observed directly by Kao Hwang(13) who stimulated the pharyngo-esophageal nerve

* Depertment of Otolaryngology and Bronchoesophagology, University of Tokyo school of Medicine Tokyo, Japan which comes off vagus nerve superiorly to the ganglion nodosum, however, this author demonstrated direct observation of esphageal contraction via esophagoscopy by stimulating the cervical portion of the vagus nerve.

It has been claimed that the cricopharyngeus muscle is a functioncl sphinctor clinically. Denervation of this muscle by cutting the vagus results in the increased tonus of the muscle and dysphagia. These studies have been done by Kirchner recently(16).

The state of tone of this muscle at rest is still not understood(20). The author tried to measure the resting potentials by means of electromyography.

Many clinician have observed that increased tonus of this muscle produces dysphagia. This is attributed to the disfunction of vagus nerve or hyperactivity of cercial sympathetic nerve ${ }^{(16)}$.

The sphincter action of the lower end of esophagus has been discussed by many scholars for a long time, however, no definite conclusions have been made(4. 19).

No anatomical sphincter has been demonstrated. Respiratory effects on the closing mechanism have not been elaborated clearly. Jackson emphasized the pinch cock mechanism of the diaphragma on the sphincter action of this part of esophagus(15).

Action potentials of the diaphragm were recognized by Agostoni, however, this was done indireetly, not by inserting a needle directly into diaphragm(2).

The author tried to measure the action 
potentials by direct method. We inserted a aneedle into the esophageal muscle layer, confirming the location of the needle point by means of a muscle identifier. This permitted clear identification of the action potentials of the esophagus and diaphragm.

Achalasia of the esophagus has been in. vestigated by many scholars for the years. Recently, a most excellent work on achalasia of the cardia by Adams and his group was done $e^{(1,22)}$. The etiology of this disease is not yet determined satisfactorilly.

Since Cannon found and wrote about "the law of denervation", (4) strong emphasis on the defect in cholinergic innervation has been considered important in the etiology of achalasia. A comparative study of this disease to Hirschsprung's disease was done by Trounce(22). Dysfunction of the plexes of Meissner and Auerbach are considered by many to be etiologically sifnificant.

Experimentally, the administration of anticholinesterase agent to animals induced achalasia in dogs. Many subsequent inveetigators confirmed these observations ${ }^{(10)}$.

Action potentials of the muscles after adminfstration of this drug were found to be increased in amplitude and frequency.

These agents are said to induce dysfunction of the neuro-muscular junction after long administration with maximum dosage. The author tried to measure normal and abnormal potentials and also tried to induce achalasia.

Long et al observed distinct difference in action of esophagus after bilateral cervical vagatomy ${ }^{(17)}$ and indued surgical achalasia by this method(18).

Congenital achalasia of the dog is observ. ed by verterinarians occasionally. This author measured the action potentials from the esophagus of such a dog.

Electromyography has been used for the diagnoagsis of lower motor neuron disease: and for other research purposes for the past: few years ${ }^{(3)}$. The author considers the fibrillation potentials, positive sharp waves and polyphasie potentials as signs of denervation. of lower moter neuron origin. On the other hand, the amplitudes and frequencies arecarefully measured.

\section{Apparatus, Equipment and Materials}

Model-P meditron made five channel electromyograph which permits simultaneous. recordings and display was used. Fourchannels are identical A-C amplifiers and preamplifiers. The last one is D-C pressure transducer. Each beam can be monitored on the other screen making it possible to introduce easily small amplitudes of theorder of 100 uv fibrillation denervation. potentials.

Viking V.U. (62) 4 track recorder wasemployed for immediate recordings to permit convenient playback.

Sinesquare wave signal generator, precision model E 310 microattenuator, and Simpson 260 adaptor were directly connected to the electromyograph for necessary calibration for same qualitive and quantita-. tive data for each time.

A Crass Camera was used for having selective tracings for photography from the prerecorded tracings. Speeds of $500 \mathrm{~mm} /$ sec and $250 \mathrm{~mm} / \mathrm{sec}$ enable one to distinguish the fibrillation potentials from normal motor units potentials of short duration were employed.

Specially devised curved monopolar electrodes were applied and inserted into the selective esophageal muscle layers by means. of a pair of foreign body forceps via various sizes of Jacksonesophagoscope.

Three dogs had their vagus nerves cut completely on one side and crushed on the other side, at the level below superior lary- 
ngeal nerve.

Two dogs were vagatomized on one side at the level below the superior laryngeal nerve, follwed by the same procedure on the other side.

One of two dogs with achalasia, 6 months old, had congenital achalasia. The other, 6 years old, had acquired achalasia with dysphagia, emesis and weight loss for 6 months.

Three cats were given Humorsoal (Demecarium bromide), one of the anticholinesterase agents, with the dosage of $0.012 \sim$ $0.02 \mathrm{mg} / \mathrm{kg}$ body weight, intramuscularly, two times a week for $4 \sim 5$ months.

Three other cats had their cervical vagus nerves severed on one side below superior laryngeal nerve, followed by the same procedure on the other side.

The last two cats were used as a control and kept and fed under the same condition as the other previously mentioned.

Nembutal was a choice of anesthesia with a dosage of $25 \mathrm{mg} / \mathrm{kg}$ body weight, intraperitoneally for cats and intravenously for dogs.

\section{Method}

Passing the suitable scope into the esophagus, an active electrode was inserted into the selective muscle layer over the scope as close as possible to the tip of the scope, at various levels $5 \mathrm{~cm}$ apart in dogs and $3 \mathrm{~cm}$ apart in cate, on each muscle layer, from the cardia up to the cricopharyngeus muscle.

By direct stimulation of muscle by a $0.01 \sim 0.1 \mathrm{~mA}$. current the location of the tip of electrode in the muscle layer was identified. The action of circular layers or longitudinal layers was clearly noted by this stimulation.

The esophagoscope itself was the inactive electrode and this made a distance between active and inactive electrodes of not more than I cm avoiding any undesirable artifacts.

With weaker anesthesia, better action potentials were obtained; the animal was well grounded before each examination.

Two channels were used; one for the esopageal muscle and the second was for the intercostal muscle to obtain respiration action potentials.

Hollinger's endoscopic camera was used to visualize and demonstrate the difference of action after stimulation, between circular and longitudinal layers osophageal muscles.

Careful anatomical dissections of vagus nerves in the neck of cats was done to trace the small fibers and branches from vagus trunks to the muscles of the esophagus with the aid of a Zeiss operative microscope.

\section{Results}

1) By the methods above, it was hard to obtain the resting potentials from normal esophagus on both layers, on both animals. 2) Spontaneous discharges from cricopharyngeus muscle of cats and dogs were obtained. Action potentials were always recorded from the posterior part of the muscle so that one could avoid the interference from cricoaryteroid posterior muscles.

Amplitudes ranged from $100 \mathrm{uV}$ to $1000 \mathrm{uV}$ with frequencies of $8-30 / \mathrm{sec}$. (Fig. 1). The action of this muscle was observed and shown by high speed endoscopic camera. Concentric contraction was observed (Fig. 2).

3) Respiratory effects were noted on the lower part of esophagus on both cats and dogs. From $1.2 \mathrm{~cm}$ below the esophgogastric junction to $2-3 \mathrm{~cm}$ above the esophgogastric junction, to $3-4 \mathrm{~cm}$ above the esophagogastric junction on dogs.

Spike action potentials were synchroni- 
Fig. 1

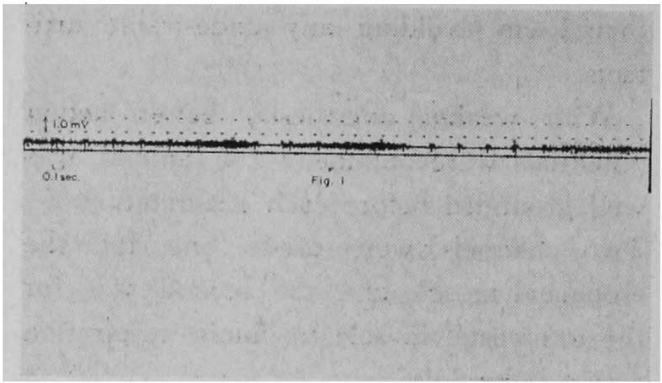

Upper beam: E.M.G. from intercostal muscls. The firing on inspiration.

Lower beam: E.M.G. from the cricopharyngeus muscls. The firings are indipendent to respiration.

Fig 2 (A)

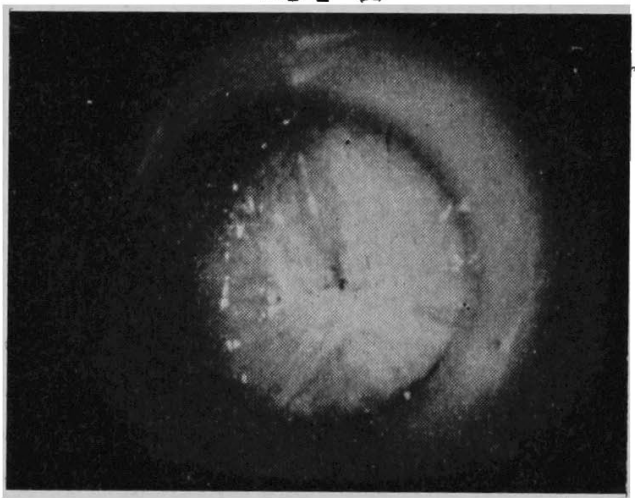

The action of cricopharyngeus muscle. Photographed by the Hollinger's camera. The musele is fully contracted.

Fig. 2 (B)

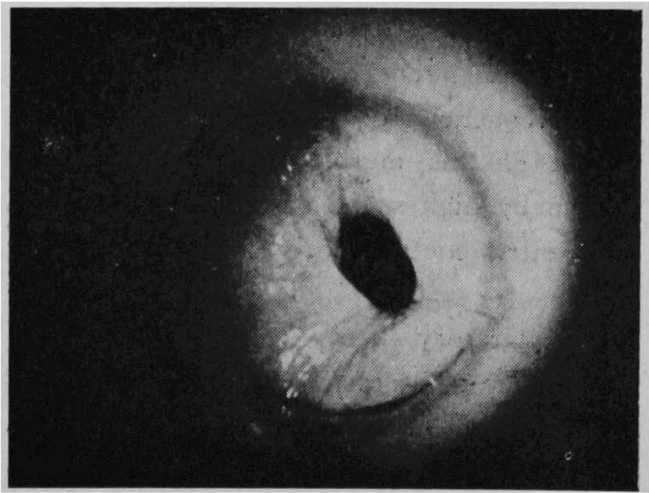

The action of cricopharyngeus musle. Ppotographed by the Hollinger's camera. The muscle is partially contracted. Both concentric and spiral actions (contraction) are observed.
Fig. 2 (C)

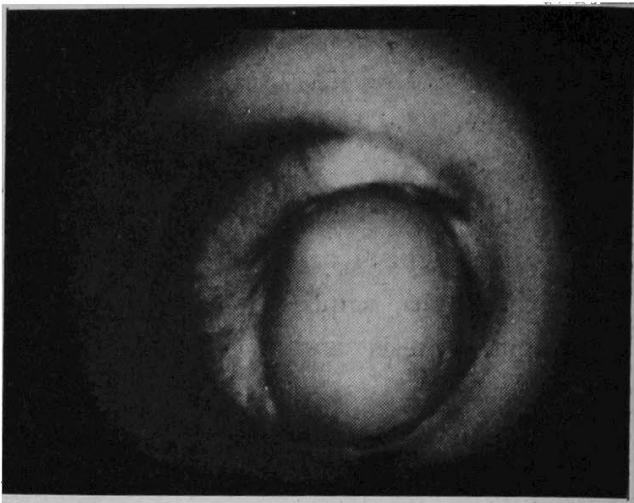

The action of cricopharyngeus muscle. Photographed by the Hollinger's camera. The muscle is fully dilated.

Fig 3

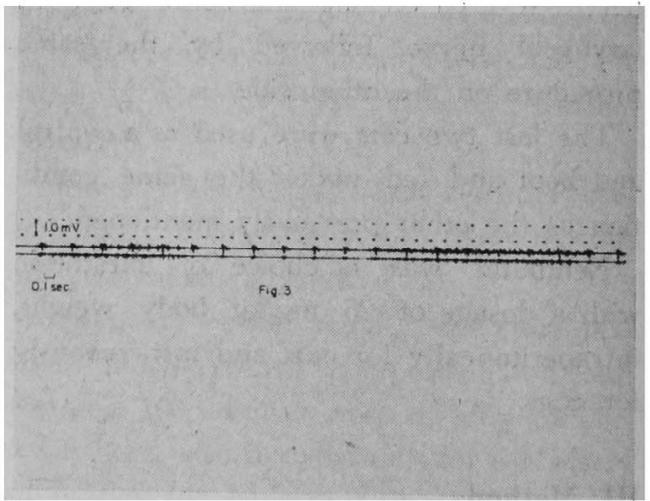

Upper beam: E.M.G. of intercostal muscle. Discharges on inspiration.

Lower beam: E.M.G. of esophageal muscle (circular layer) $3 \mathrm{~cm}$ above G. E. jun. ction.

Discharges synchronize to inspiration.

zed with each inspiratory phase and equally noted on circular layer and longitudinal layer (Fig. 3). No respiratory effects were seen on the middle and upper parts of esophagus. These spike potentials are not confused with diaphragmatic potentials because, the needles were known to be (see experiment method) in the esophageal muscle layer.

4) To identify the muscle layer to be 
Fig. 4

Dog 83, $15 \mathrm{kgm}$. 2 weeke after unilateral Cervical Vagotomy (LT.)

\begin{tabular}{|c|c|c|c|c|c|c|c|c|c|}
\hline \multirow{3}{*}{$\frac{20 \mathrm{~cm} .}{(\text { Cricophar. M.) }}$} & \multicolumn{2}{|c|}{ Anterior } & \multicolumn{2}{|c|}{ Posterior } & \multicolumn{2}{|c|}{ Left } & \multicolumn{2}{|c|}{ Right } & \multirow{2}{*}{ Remarks } \\
\hline & \multicolumn{2}{|c|}{ Cir. Long } & \multicolumn{2}{|c|}{ Cir. Long } & \multicolumn{2}{|c|}{ Cir. Long } & \multicolumn{2}{|c|}{ Cir. Long } & \\
\hline & \multicolumn{2}{|c|}{ W } & \multicolumn{2}{|c|}{ HH } & \multicolumn{2}{|c|}{$\because \quad H$} & \multicolumn{2}{|c|}{ WI } & $\begin{array}{l}\text { All normal motor unit poten- } \\
\text { tials }\end{array}$ \\
\hline $25 \mathrm{~cm}$ & - & - & - & - & - & - & - & - & \\
\hline $28 \mathrm{~cm}$. & Ht & H & - & - & $H$ & + & - & - & $\begin{array}{l}\text { All fibrillation volages with } \\
\text { possharp potential }\end{array}$ \\
\hline $30 \mathrm{~cm}$. & 詶 & $H$ & \# & 性 & $H$ & + & H & + & $\begin{array}{l}\text { Fibrillation with pos. sharp } \\
\text { potential; normal motor unit }\end{array}$ \\
\hline $33 \mathrm{~cm}$. & 册 & $H$ & IH & H & mt & $H$ & 册 & $H$ & Same above \\
\hline $35 \mathrm{~cm}$. & m & I H & H & H & m & $H$ & m & H & Same above \\
\hline $38 \mathrm{~cm}$. & 4 & tit & \# & H & m & H & 册 & H & Same above \\
\hline $40 \mathrm{~cm}$. & + & + & + & + & H & + & $\mathrm{H}$ & . H & Same above \\
\hline $43 \mathrm{~cm}$. & H & $!-$ & H & - & H & - & + & + & Same above \\
\hline $45 \mathrm{~cm}$. & - & -- & - & - & - & - & + & - & All fibrilltion potentials \\
\hline $\begin{array}{c}47 \mathrm{~cm} . \\
(\mathrm{G}-\mathrm{E} \text { junction) }\end{array}$ & - & - & - & - & - & - & - & - & \\
\hline
\end{tabular}

examined, greater amouts of amperes were needed to get satisfactory contraction of muscle on unilaterally vagotomized animals.

Fibrillation denervation potentials, positive denervation potentials and polyphasic potentials, which are clearly seen in the denervated skeletal muscles usually, were shown on every side of esophageal wall and in both layers, eight to tan days after the dogs and ten to fourteen days after the cats had unilateral cervical vagotomy (Fig. 4).

With the dogs, these abnormal potentials were seen anywhere from $2.5 \mathrm{~cm}$ above the esophagogastric junction or $45 \mathrm{~cm}$ below incisor up to $25 \mathrm{~cm}$ below incisor or $5 \mathrm{~cm}$ below cricopharyngeus muscle (Fig. 4).

On the cats, these fibrillation denervation patentials were picked up from $30 \mathrm{~cm}$ below incisor on the beginning of the cardia up to $15 \mathrm{~cm}$ below incisor or $5.0 \mathrm{~cm}$ below cricopharyngeus muscle. Low $1 / 5$ of esophagus consists of smooth muscle and other of striated muscle in cate (Fig. 5, chart).

\section{Fig. 5}

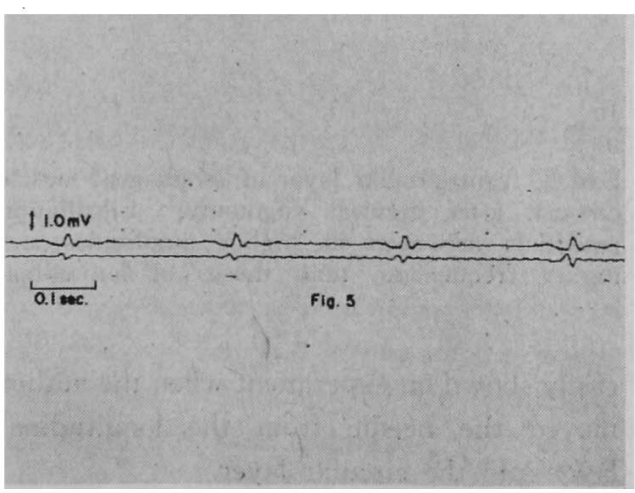

Upper beam: E.M.G. from lower part of esophagus. Marked fibrillation potentials are seen.

Lower beam: E.M.G. from inter costal muscle, on silent stage. 
Action potentials were found in increased frequeney and higher amplitude in circular layer than longitudinal layer of muscles on both animals (Fig. 6, tracings). This is

Fig. 6 (A)

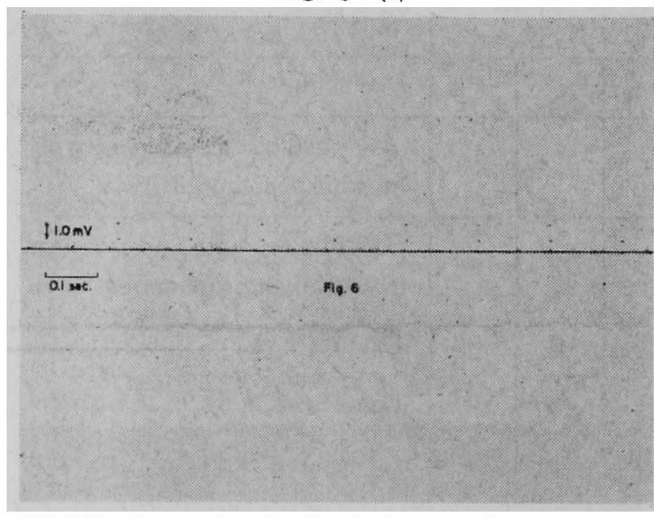

E.M.G. from longitudinal layer of esophageal muscle on cat, after cervical vagotomy. Fibrillation potentials are seen.

Fig. 6 (B)

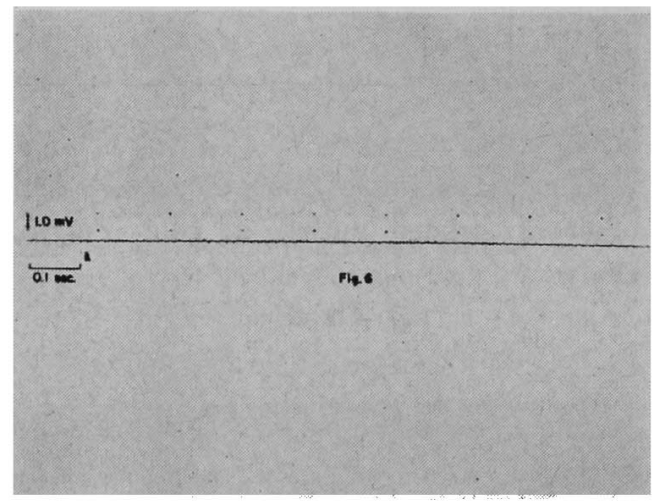

E.M.G. from circular layer of esophageal muscle on cat, after cervical vagotomy. Fibrillation potentials are seen in higher amplitudes and higher frequencies, than thoses of lonitudinal layer.

easily shown on experiment when the author moved the needle from the longitudinal layer into the circular layer.

Signs of regeneration were seen 9 weeks after unilateral vagotomy on dogs and 12 weeks on cats, by recognition of giant motor mit potentials, nacent motor unit potentials, and polyphasic potentials. Respiratory effects on these esophagi were observed besides spontaneous denervation voltages, and these were found more active in frequencies and amplitudes than these of normal intact animals. No denervation potentiale were measured from cricopharyngeus mucle.

5) These studies were followed by cervical vagotomy on the other side, which permitted the animals to live only 5-7 days, 10 weeks after first vagotomy on dogs and 2832 weeks on cats.

Frequencies and ampli- tudes of action potentials found in circular laye $\mathrm{I}$ were much higher than in longitudinal layer in both animals.

Respiratory effect on the denervated esophagus was observed, besides the spontaneous denervation potentials. No denervation potentials were recorded from circopharyngeus muscle after bilateral vagotomy.

Direct visualization of lower part of esophagus showed the evidence of contraction of lumen and dilatation of middle part by bilateral vagotomy on 2 of the 3 cats.

6) It is interesting to note that definite fibrillation potentials and positive sharp waves were seen at the lower end of esophagus of cats after bilateral and unilateral vagotomy (Fig. 7). This part proved consist of smooth muscle entirely, by histological study.

Denervation potentials were not seen in lower part of esophague of the dogs inspite of the presence of striated muscles.

7) Electromyographic findings on achalasia dogs were quite interesting. One dog was a 6 months old Irishsetter with congenital achalasia (Fig. 8, X-ray). The entire length of esophagus was $45 \mathrm{~cm}$ below incisor and lower $5 \mathrm{~cm}$ was constricted. The other was a 7 year old Newfoundlander with acquired achalasia with persistent dysphasia, emesis 
Fig. 7

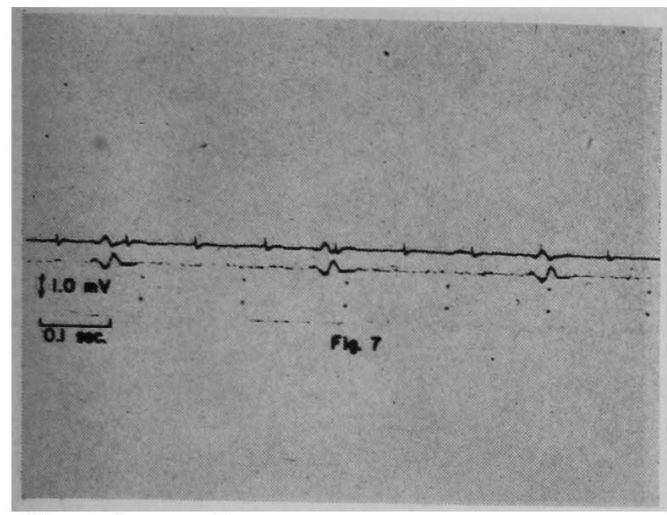

Upper beaw: E.M.G. from lower end of esophagus of cat after cervical vagotomy. Definite positive sharp wave potentials are seen. Lower beam: E.M.G. from inter costal muscle on silent stage.

Fig. 8

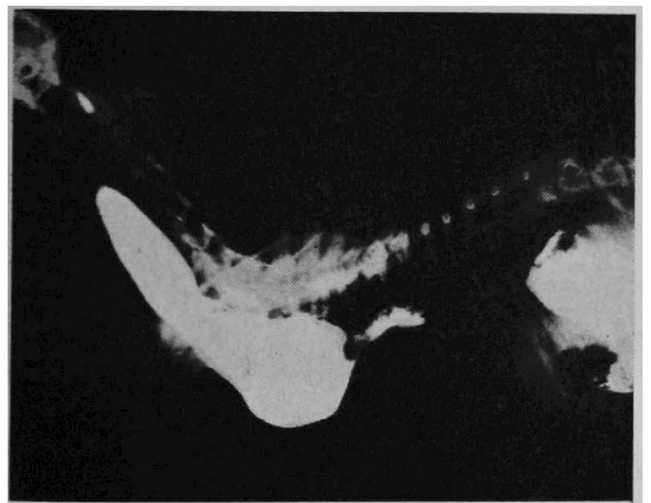

x-ray film of 6 mos. old dog with achalasis (congenital) E.M.G. from the esophagus of this dog is shom in Fig. 9.

and a prolonged deglutition time for 6 months. The entire length of esophagus was $53 \mathrm{~cm}$ from incisor and it had dilated all the way to the cardia. Electromyographic studies were done in same manner under nembutal anesthesia.

On the former dog, a tremendous amount of fibrillation denervation potentials and positive sharp posentials were picked up from the dilated part of the esophagus at any level and site, especially from the low-
Fig. 9

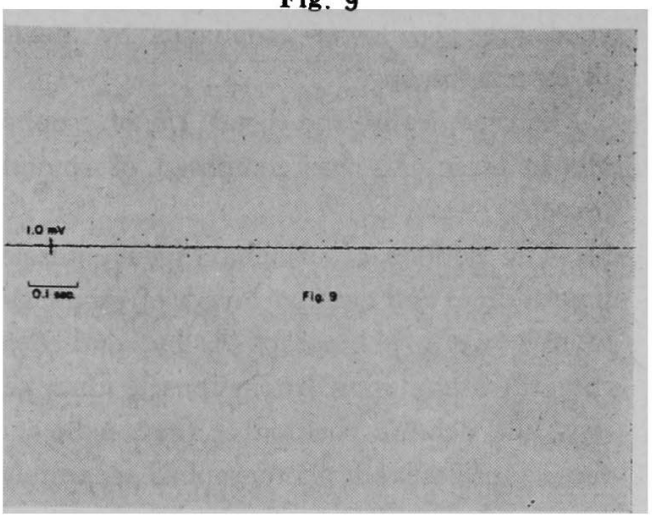

E.M.G. of the achalasia dog. Marked fibrillation potentials are recorded from the dilated part of this esphagus (see Fig. 9).

er part of dilation (Fig. 9). No fibrillation potentials were obtained from the cardia This represents the severance of lower motor neurons which innervate the dilated part of esophagus, by any cause, in any part.

On the latter dog, no fibrillation potentials were seen. But, normal motor units were obtained from the dilated portion of the esophagus. This was more apparent at the level of $40-45 \mathrm{~cm}$. On both dogs, more electrical activity, both normal types, were seen in the distal areas of dilation.

8) For the purpose of induction of achalasia, humorsol was given to 3 cats. One hundred days after drug (humorsol) administration, fibrillation denervation potentials with positive sharp waves were obtained from the esophagus and intercostal muscle of cats.

Atropine was necessary to compensate for the overaction of cholinergic effects such as hypersecretion and edema of the tissue, particularly pulmonary edema. Humorsol administration was stopped at the end of the 5th month because the animals were becoming weak.

Three cats showed some evidence of achalasia 13 weeks after administration started. This was observed as fairly dilated part 


\section{気食会報一16 卷}

on middle and lower esophagus and tightened area on lower esophagus by means of esophagoscope.

Microscopically, the distal $1 / 5$ of esophagus in these cats was composed of smooth muscle.

9) The actions of criopharyngeus muscle, longitudinal and circular layers of esophagel muscle were photographed by endscopic camera. Stimulation by the muscle identifier gave the definite position of the needle and these muscles were photographed separately. Electrical stimulation of vagus nerve on one

Fig. 10 (A)

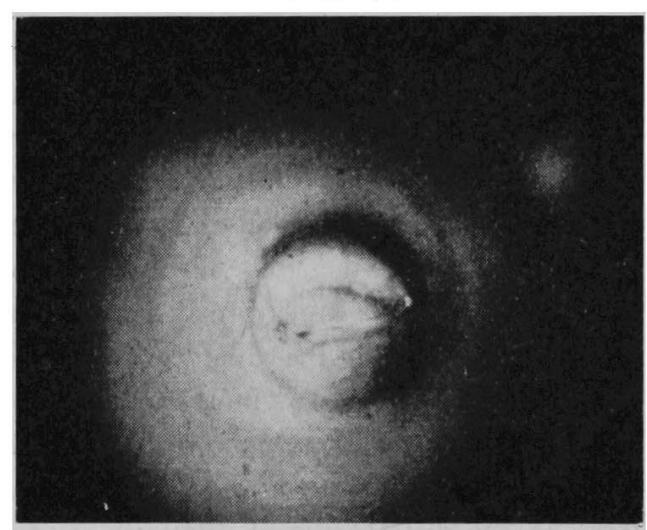

The action of esophagus by the electrical stimuJation of the cervical vagus nerve.

Fig. $10 \quad$ (B)

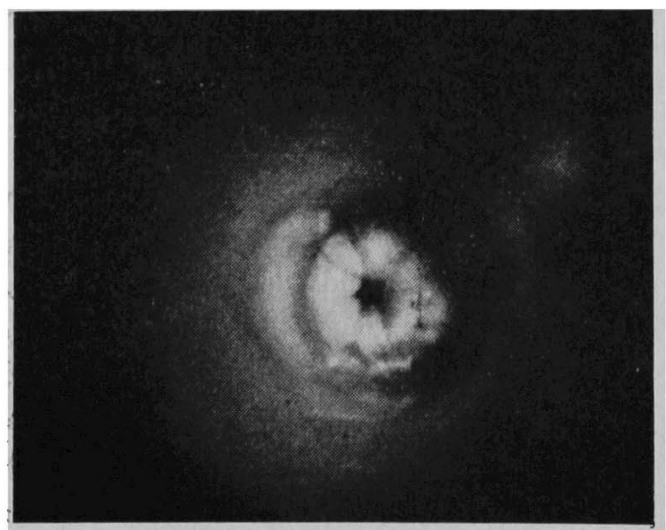

The action of circular layer of the esophageal muscles by electrical stimilation.
Fig. 10 (C)

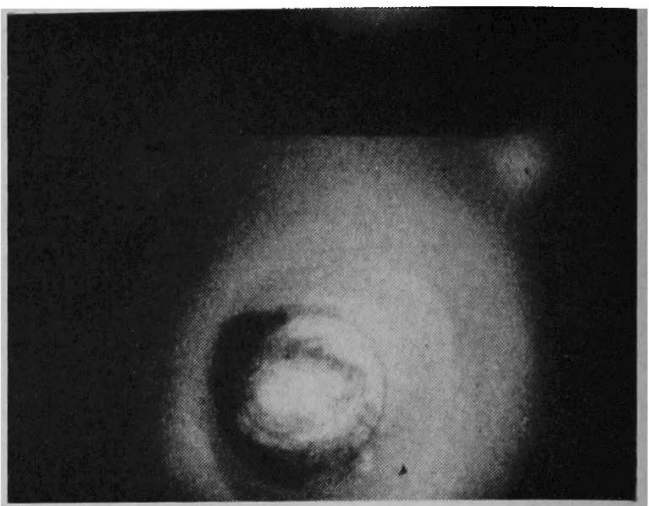

The action of longitudinal layer of the esophageal muscles by electrical stimulation.

Fig. 11

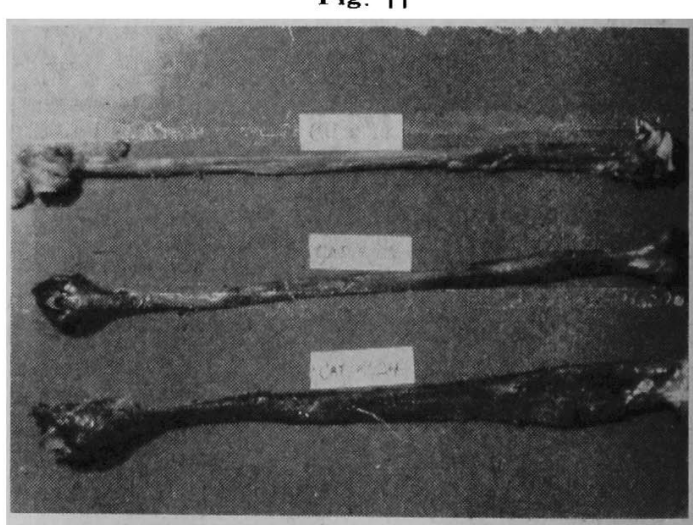

Cat $\# 11$ :

Dissected esophagus after unilateral cervical vagotomy. No evidence of dilatation.

Cat $\# 9$ :

Dissected esophagus after long term administration of humorsol. Moderate dilatation on lower esophagus.

Cat $\# 24$ :

Dissected esophagus after bilateral cervical lägotomy. Marked dilation of mid-lower esophagus.

side at the level of thyroid cartilage caused contraction of the entire esophagus. This was observed, by an endoscopic camera (Fig. 10).

10) Autopay of animals was performed following the above studies. Unilateral cervical vagotomy case showed no evidence of achalasia. Humorsol case showed some 
light evidence of dilatation of esophagus on its lower part, but not at esophagogastric junction. The case with bilateral cervical vagotomy ahowed marked evidence of dilation of esophagus on its middle and lower portion including esophagogastric junction (Fig. 11).

\section{Discussion}

1) Lerche(18) described the muscular layer of human esophagus macroscopically and microscopically. The cat esophagus is the most similar to the human esophagus among domestic experimental animals from the histological point of view. Because dog esophagus consists entirely of striated muscle, cat esophagus is the most similax to human for measuring the normal and abnoral action potentials.

2) After careful dissection of cats and dogs under an operative microscope, only small fibers innervating the esophagus from the vagus trunk were seen excepting for the branches from the pharyngeal plexes to the apper portion of cervical esophagus. This means that even slight pathology around these fibere may cause the damage to the fibere and partial denervation of esophagus, such as was seen with the achalasia dog by the electromyogram which showed fibrillation denervation potentials. Further studies are necessary for the investigation of denervation of esophageal musculature.

3) Equality of denervation potentials from every side of esoohagus after unilateral cervical vagotomy indicates that innervation of esophagus is bilateral; hence, unilateral denervation does not cause achalasia experimentally.

4) Synchrconized action potentials to respiration have been recorded from lower part of esophagus on both dogs and cats in spite of histological differences. No apparent respiratory effects on the middle and upper parts of esophagus were seen. 5) Fibrillation denervation potentiale from the cardia, which consists of only smooth muscle in the cat, should be investigated more: since the experimental data indicates their presence contrary to prior work.

6) The difference of activity between longitudinal and circular layers is very interesting and this was investigated by means of the classical kimograph by Eura in $1937^{(9)}$ and pharmacologically by Ellis in $1960^{(8)}$.

Electromyography clearly showed the difference of electrical activity between both layers.

All the cats and dogs showed these phenomena and the author assumes this applies to the human esophagus. The difference of activity between these muscle layers and dominance of the circular layer is probably aitributed to any functional disorder of the esophagus such as, Schatzki's ring, ftnctional constriction of esophagus. such as tertiary contraction or tired spasm, achalasia and other functional dysphagia, when the functional balance of these muscles is broken.

7) Long term administration of an anticholinesterase agent to the animals results in constant acetylcholine transmission in neuromuscular junction, thus destroying many myenteric and submucous plexes. This is one way to induce the achalasia, and has been proved histologically.

Electromyographic findings at this point are of great interest. The fibrillation denervation potentials were recorded from various parts of skeletal muscles of the animal as well as from esophageal muscles.

The etiology of achalasia is still unknown. Wether dysfunction of the plexes or diseased lower motor neurons play apart should be investigated further by using electromyography.

8) Spontaneous electrical discharges from 
cricopharyngeus muscle were obtained.

The tonus of this muscle is higher than that of the other part of dsophageal muscles at resting stage.

9) Autopay of animals demonstrated distinctly that achalaasia was induced in animals by the methods mentioned above, and that upper part of esophagus must have innervation from superior laryngeal nerve and pharyngoesophageal nerve (Hwang) (12) and some other branches because there is no evidence of dilation after vagotomy.

\section{Conclusion and Summary}

1) Seven dogs including two achalasia dogs and seven cats were studied by electromyography of esophagus.

2) Spontaneous discharges from the cricopharyngeus muscle were recorded.

3) The difference in action potentials between longitudinal and circular layers of esophagus was observed, and the dominance of the circular layer was noted.

4) Fibrillation denervation potentials were recorded from the dilated part of achalasia esophagus of the dog.

5) Findings indicate one possible etiology of achalasia is damage of lower motor neurons.

6) Fibrillation denervation potentials were recorded from the muscles of animals after long term administration of anticholinesterase agent

7) The innervation of esophagus is bilateral.

8) Electromyography is established as another method of investigating of the pathophysiology of the esophagus.

1) Adams, C. W., Maples, E. A., Trounce, J. R.: Achalasia of the cardia and Hirschsprung's disease. The amount and distribution of cholinesterase.

Clin. Sci., 19: 478-481, 1960.

2) Agostoni, E., Santanbrogio, G.: Electromyography of the diaphragm in man and transdiaphragmatic pressure.
J. of Applied Physiol., 15: 1093-1097, 1960.

3) Buchthal, F.: An introduction to electromyography.

4) Cannon, W. B.: A law of denervation. Am. J. Med. Sci., 198: 737, 1939.

5) Clark, C. G., Vane, J. R.: The cardiac sphincter in the cat.

GUT. 2: 252-262, 1961.

6) Code, C.: An atlas of esophageal mobility in health and disease. by Charles Thomas publisher, 1958.

7) Doty, R., Bosma, J, R.: An electromyographic analysis of reflex deglutition. J. Neurophysiology, 19: 44-60, 1956.

8) Ellis, F. G., Trouce, J. R.: The innervation of the cardia and lower esophagus in man.

Brit. J. Surg., 47: 466-472, 1960.

9) Eura, S.: Studien über die Muskulatur des Oesophagus Vershiedener Tiere.

JAP J. Med. Sci., TRIII, Biophysics, 1: $1-51,1927$.

10) Harris, L. D., Ingelfinger, F. J.: Esophageal peristalsis and achalasia produced in dogs by prolonged cholinesterase inhibition. J. Clin. Invest., 39: 1744-1757, 1960.

11) Hiroto, I.: Physiology and pathophysiology of esophagus.

Ann. Broncho-esoph. JAP, 7: 1-19, 1958.

12) Hwang-Kao: Mechanism of the esophagus after bilateral resection of the pharyng. oesophageal nerve in the dog.

Am. J. of Physiol., 174: 231-234, 1953.

13) Hwang-Kao: Nervous control of the cervical portion of the esophagus.

Am. J. of Physil., 154: 343-357, 1948.

14) Hwang-Kao: Mechanism of transportation of the content of the esophagus.

J. of Applied Physiol. 6: 781-796, 1954.

15) Jackson, D.: Diaphragmatic pinchock in socalled cardiospasm.

Laryngoscope, 32: 139, 1922.

16) Kirchner, J. A.: The motor activity of the cricopharyngeus muscle.

Laryngoscope' 118: 1119-1159, 1958.

17) Lerche, W.: Esophagus and pharynx in action.

Chrles Thomas Pudlisher, 1950

18) Long, D. M., Nice: The experimental production of cardiospasm in dogs.

Surg. Form., 9: 408-411, 1958.

19) Schenck, E. A.: Cardiac and cricopharyngeal sphincter thresholds in cats.

Am. J. of Physiol., 197: 743-746, 1959.

20) Teracol: Diseases of the esophagus. Saunders, publishers.

21) Trounce, J. R., Dencker, D. C., Kauntzo, R., Thomas, G. A.: Studies in achalasia of the cardia.

Quart. J. Med. 26: 433, 1957. 